\title{
Indications d'avulsion des dents de sagesse mandibulaires dans le cadre militaire
}

\author{
Indications for the removal of mandibular third molars in a military context
}

\author{
MATHIEU GUNEPIN¹, FLORENCE DERACHE², BENOÎT BEDRUNE³ ${ }^{3}$ YVAN FRONTY ${ }^{4}$
}

\begin{abstract}
RÉSUMÉ
La décision d'extraire ou non des dents de sagesse mandibulaires, notamment asymptomatiques, est souvent mal comprise par les personnels militaires. Cet acte chirurgical est perçu comme systématique et allant à l'encontre des recommandations des praticiens civils.

L'objectif de ce travail est de mettre en exergue le fait que les décisions des praticiens militaires se prennent conformément aux recommandations de bonnes pratiques civiles avec une évaluation du rapport bénéfices/risques de l'intervention pour les patients. Cependant, les services de santé des armées, français et étrangers, doivent impérativement prendre en considération certains paramètres inhérents aux conditions de vie des militaires, notamment au cours des opérations extérieures, afin d'orienter les choix thérapeutiques dans le but de maintenir la capacité opérationnelle des Forces. (Med Buccale Chir Buccale 2008 ; 14: 193-208).
\end{abstract}

mots clés: armée, avulsion, dent de sagesse

\section{SUMMARY}

The removal of mandibular third molars is frequent in French and foreign armies. This doesn't mean that theses extractions are systematic for all soldiers. The military practitioners follow the same recommendations than their civilian colleagues by establishing a ratio benefits/risks for their patients.

In the civilian area, the consequences of the surgical approach (extractions of third molars) are well known, but the benefits of the conservative approach are not well documented (few number of studies, biais in the constitution of groups of patients... ). This is why the indications for the removal of mandibular third molars are not always easy to take by civilian practitioners.

In the army, other parameters are taken into account. The military conditions of life, especially during operations, enforce a particular ratio benefits/risks for soldiers. In fact, military dentists take operations particularities into consideration: tiredness, stress, boredom sometimes lead to decrease the oral/food/personal hygiene of soldiers. All conditions are gathered to increase mandibular third molars problems (pericoronatis for example). These pathologies are a great handicap for soldiers but also for all Units. A medical evacuation on a battle field generates a lack of operational capabilities (medical team, escort, vehicles, helicopter...) and a real risk for soldiers (improvised explosive device, road accident...). All these military particularities and their dental consequences are published through international medical journals and impact directly the indications for the removal of mandibular third molars in the military context because of the obligation of maintaining a high operational readiness. (Med Buccale Chir Buccale 2008 ; 14 : 193-208).

key words: army, removal, wisdom teeth

1 Adjoint au Chef du Secteur dentaire inter-armées Draguignan

2 Adjoint au Chef de Service de Chirurgie maxillo-faciale et Stomatologie de I'HIA Sainte Anne Toulon

3 Chef de Service de Chirurgie maxillo-faciale et Stomatologie de I'HIA Sainte Anne Toulon

4 Adjoint au Chef de Service d'Odontologie de l'HIA Robert Piqué Bordeaux

Demande de tirés à part:

Mathieu Gunepin Secteur dentaire inter-armées BP 40083007 Draguignan Cedex mgunepin@yahoo.fr 
médecine

buccale

chirurgie

buccale

VOL. $14, \mathrm{~N}^{\circ} 4$ 2008

page 194
La décision d'extraire ou non une dent de sagesse mandibulaire est souvent difficile à prendre. En effet, dans la cadre de leur pratique quotidienne, les chirurgiens dentistes civils et militaires, français ou étrangers, se doivent de baser leurs actes sur des faits avérés dans le respect de l'odontologie fondée sur la preuve (evidence based dentistry ${ }^{[1]}$ ). Or, dans le domaine de l'avulsion des dents de sagesse, les publications foisonnent, mais les conclusions de la littérature sont toujours prudentes et ne permettent pas aux praticiens de fonder leurs indications sur une argumentation scientifique.

La prise de décision incombe donc souvent au praticien seul qui va devoir se baser sur l'évaluation du rapport bénéfices/risques de l'extraction d'une troisième molaire chez son patient. Toute la difficulté réside dans le fait que si les risques liés à la chirurgie sont bien identifiés, il n'en est pas de même pour les bénéfices inhérents à la conservation ou non d'une dent de sagesse. Cette constatation fait d'ailleurs l'objet d'un large consensus. Dans ce cas, comment les praticiens peuvent-ils prendre leur décision alors qu'ils n'ont pas tous les paramètres en main ?

Dans le milieu militaire, les chirurgiens dentistes sont largement aidés dans le cadre de leurs prises de décision d'avulsion de dents de sagesse par les nombreuses publications, notamment anglosaxonnes, portant sur les retours d'expérience d'opérations extérieures et plus largement sur l'analyse de l'impact des pathologies bucco-dentaires sur les capacités opérationnelles des personnels en mission. Ainsi, le rapport bénéfices/risques en milieu militaire est différent du milieu civil compte tenu du contexte opérationnel et de ses impératifs.

A travers une revue de la littérature internationale, nous nous intéresserons à l'approche civile de la décision d'extraction des dents de sagesse mandibulaires, puis nous verrons que le milieu militaire implique forcément des spécificités qui ont conduit certaines Armées (américaine, canadienne, anglaise, australienne...) à publier des textes réglementaires clairs et argumentés en matière de prise en charge des dents de sagesse avec toujours pour objectif de maintenir la capacité opérationnelle des Forces armées sur les théâtres d'opérations extérieures.

\section{RAPPELS}

\section{Mise en place des dents de sagesse mandibulaires}

Les troisièmes molaires, plus communément appelées dents de sagesse, sont les seules dents qui se développent entièrement après la naissance ${ }^{[2]}$ (début de formation à partir de l'âge de 5 ans ${ }^{[3]}$ ). Leur développement est intimement lié à celui de leur environnement et notamment à celui de la deuxième molaire.

Les deuxièmes et troisièmes molaires, après la formation de leur couronne, migrent progressivement vers la gencive en se verticalisant au contact de la face distale de la molaire précédente, décrivant la classique « courbe de Capdepont » ${ }^{4]}$.

Le premier écueil à une éruption verticale en bonne position des dents de sagesse est le manque de place sur l'arcade pour l'évolution de la dent. Les dents de sagesse mandibulaires peuvent ainsi être retenues sous le bord antérieur de la branche montante par insuffisance de l'espace rétromolaire. De plus, ces dents se mettent en place à un moment où l'os mandibulaire est mature, elles vont donc devoir traverser un os particulièrement compact dans cette région.

Le deuxième obstacle à la verticalisation complète des dents de sagesse mandibulaires, même avec un espace rétromolaire suffisant, est un redressement d'axe trop important pour les dents en formation. De ce fait, les dents n'achèvent pas leur trajet et leur couronne reste enclavée sous le collet de la dent de 12 ans (deuxième molaire). De plus, à une obliquité potentiellement défavorable du germe, se surajoute le développement postérieur de l'arc mandibulaire qui entraîne également vers l'arrière l'ébauche des racines en les incurvant. En fait, ces deux étiologies (manque de place, défaut de verticalisation) paraissent le plus souvent s'imbriquer.

Cependant, les raisons de l'apparition de malpositions des dents de sagesse et les mécanismes qui les contrôlent restent encore largement méconnus [5]. 


\section{Epidémiologie}

Un volet épidémiologique est indispensable à la compréhension de l'intérêt suscité par les dents de sagesse auprès de la communauté scientifique et médicale. Cet intérêt se traduit au fil des années par la multiplication des publications, par la diffusion de règles de bonnes pratiques professionnelles et par des conférences dites de consensus [6-12].

Si l'inclusion des dents de sagesse n'est pas une nouveauté, l'augmentation de la fréquence de ces inclusions semble récente. Le cas le plus ancien d'inclusion d'une dent de sagesse mandibulaire a été découvert en 1911 sur le squelette de la Magdalenian Girl âgée de 13.000 à 15.000 ans [13], qui avait entre 25 et 35 ans au moment de son décès. Chez l'homme moderne, les troisièmes molaires sont des dents particulières. Elles ont le plus fort taux de polymorphisme, de malposition sur les arcades, d'inclusion et d'agénésie [14, 15]. En France, sur un échantillon de 1550 cas, Buisson trouve une fréquence d'inclusion, de rétention ou d'enclavement de la troisième molaire mandibulaire de l'ordre 58,5\% [16]. Dans les recommandations de l'ANAES de 1997, il est noté que $10 \%$ de la population française a au moins une dent de sagesse incluse, retenue ou enclavée, et que 15 à $25 \%$ des troisièmes molaires mandibulaires sont incluses, retenues ou enclavées [17].

Dans la littérature étrangère, Sands et coll. ${ }^{[18]}$ soulignent qu'environ $65 \%$ de la population humaine a au moins une dent incluse à l'âge de 20 ans, principalement des dents de sagesse mandibulaires. Hugoson et coll. affirment que l'inclusion de la troisième molaire est fréquente puisque $72,7 \%$ des suédois âgés de 20 à 30 ans ont au moins une troisième molaire incluse ou enclavée (73 \% des 20-29 ans selon Crosse [19]) alors que ce pourcentage était de $20 \%$ dans les années 1930 [20]. Pour les dents de sagesse mandibulaires, Bjork et coll. constatent que sur un échantillon de 237 étudiants suédois de sexe masculin âgés de 19 à 30 ans [21], seules $38 \%$ des dents de sagesse mandibulaires sont en place sur l'arcade. Cette forte prévalence de l'inclusion de la troisième molaire mandibulaire est confirmée par
Stanley et coll. qui, après l'examen de 11598 radiographies panoramiques dentaires de patients de plus de 20 ans, observent que $15,1 \%$ des patients avaient une dent incluse, retenue ou enclavée (quel que soit le type de dents) et que parmi l'ensemble des dents incluses, la prévalence des troisièmes molaires mandibulaires était de $55,9 \%$ [22].

Si la fréquence d'inclusion des dents de sagesse en général et des dents de sagesse mandibulaires en particulier est élevée, celle de leur extraction l'est aussi. Près de $90 \%$ des personnes inscrites sur les listes d'attente des services de chirurgie buccale et maxillo-faciale en Angleterre et au Pays de Galles attendent l'avulsion de leurs dents de sagesse [23]. L'extraction des dents de sagesse est d'ailleurs décrite par Sands et coll. comme la procédure opératoire la plus pratiquée en chirurgie buccale et maxillo-faciale [24]. En France, les avulsions de dents de sagesse incluses représentent $63 \%$ des interventions de chirurgie buccale traditionnelle avec hospitalisation [25].

\section{APPROCHE CIVILE}

\section{Evaluation du rapport bénéfices/ risques encourus}

Comme pour tout acte chirurgical, la réflexion qui mène à proposer l'intervention devrait tenir compte du rapport bénéfices/risques encourus pour le patient ${ }^{[26,27]}$. Le risque chirurgical doit être inférieur aux complications potentielles associées à la conservation de la dent de sagesse [28, 29].

\section{Risques liés à l'avulsion des dents de sagesse mandibulaires}

Il est tout d'abord évident que tout acte médical, même bien conduit, recèle un risque de complications [30, 31]. Bramley [32] indique que 1 patient sur 20 connaît des complications durant ou après l'extraction de ses dents de sagesse. Selon Sands et coll. [18], ce taux de complications est de $18 \%$ au cours de l'intervention et de $20 \%$ après. Quant à Moghadam et coll., ils estiment que le taux global de complications associées à l'avulsion des troisièmes molaires est de 7 à $10 \%$ [33]. Le risque médecine

buccale

chirurgie buccale

VOL. $14, N^{\circ} 4$ 2008

page 195 
médecine

buccale

chirurgie

buccale

VOL. $14, \mathrm{~N}^{\circ} 4$ 2008

page 196 et l'importance des complications varient en fonction de l'opérateur et de la technique utilisée [34]. Ils augmentent avec la profondeur de l'inclusion et la difficulté opératoire [35], d'où l'importance des examens radiographiques pré-opératoires [36, 37]. Ces complications sont le plus souvent mineures et passagères. Elles sont d'autant plus importantes que le patient est âgé de plus de 25 ans. Le Royal College of Surgeons of England s'est intéressé aux risques liés à la chirurgie des dents de sagesse (hors risques liés aux anesthésies locale et générale) dans un guide clinique publié en 1997 et encore en vigueur [10, 12]. Ce guide fait notamment état de la description des complications en indiquant certaines fréquences :

- Complications immédiates :

- dans 1 cas sur 100, le saignement est difficile à arrêter (hémorragie) ;

- dans 1 cas sur 100, une dent autre que la dent de sagesse peut être endommagée lors de l'intervention (fracture dentaire, descellement de prothèse qui peut être inhalée ou ingérée) ;

- fracture de l'angle mandibulaire ;

- plaies par instruments ;

- fracture d'un instrument qui peut être inhalé ou ingéré ;

- dans 5 cas sur 100, un fragment radiculaire est laissé en place dans l'alvéole [38].

- Complications médiates ou retardées :

- douleurs apparaissant généralement entre 3 et 6 heures après l'intervention et disparaissant normalement après 5 à 7 jours [39] ;

- hémorragie secondaire à l'avulsion ;

- Chi et coll. ${ }^{[40]}$ et Figueiredo et coll. [41] indiquent une fréquence de survenue d'infections après l'extraction de dents de sagesse de 1 à 2 cas sur 100 extractions ;

- dans 1 cas sur 10 à 1 sur 100 , le patient peut ressentir une douleur pulsatile, liée le plus souvent à une alvéolite (dry socket), assertion confirmée par Haug et coll. [42]. Larsen ajoute que ces alvéolites sont plus fréquentes chez les patients fumeurs [43]. Le cycle menstruel de la femme a également un impact sur la fréquence des alvéolites ${ }^{[44]}$;

- l'extraction d'une dent de sagesse peut entrấner l'apparition d'une poche parodontale distale à la deuxième molaire ${ }^{[45,46]}$;
- survenue de troubles neurologiques [47-49]. Chez 1 patient sur 100 , on note une paresthésie ou une dysesthésie permanente de la lèvre inférieure, des joues et de la langue [50] et 5 à $15 \%$ des patients souffrent des mêmes symptômes au niveau des deux tiers antérieurs de la langue et de la lèvre. Schutz-Mosgau et Reich indiquent que 1 à 8 patients sur 100 peuvent avoir une "sensation de fourmillement ou d'engourdissement » de la lèvre inférieure, des joues et de la langue [51]. Queral-Godoy et coll. précisent que la plupart du temps ces troubles neurologiques sont temporaires et que leur régression se fait en trois mois [52]. II est certain que l'avulsion de la troisième molaire mandibulaire fait courir le risque d'une lésion du nerf mandibulaire (de 1,3 à 7,8 \% des cas selon Rood [53]). La prévalence des lésions du nerf lors de l'extraction de dents de sagesse radiologiquement contiguës au nerf mandibulaire est de $35 \%$ pour Commissionat [54] et de $30 \%$ pour Rood [55]. II est à noter qu'il est cependant possible de laisser en place les apex qui jouxtent le canal mandibulaire, les suites opératoires sont dans ce cas moins douloureuses et plus simples que les suites opératoires d'une extraction complète ${ }^{[54,56]}$. Robinson et coll. affirment que le nerf lingual peut être endommagé chez près d'1 patient sur 100 (0,8 \% des cas) de manière définitive [57]. Notons également la possibilité de survenue d'hyperesthésie (névralgie). La fréquence de complications nerveuses est d'autant plus élevée que la dent est profondément incluse, par contre le risque est le même que les dents soient symptomatiques ou non. Ces lésions neurologiques constituent pour le sujet concerné une véritable atteinte à son intégrité physique et psychologique, modifiant la perception de son schéma corporel [58].

Andreasen et Hakansson [59] rappellent une évidence qu'il faut bien garder à l'esprit : l'extraction dentaire est un geste irréversible alors que la troisième molaire garde tout son intérêt par exemple comme transplant afin de compenser l'absence d'une autre dent. Tous les travaux dans le domaine de la génétique, notamment visant à faire "pousser des dents », n'ont jusqu'à présent pas abouti [60]. 


\section{Bénéfices}

\section{Les bénéfices liés à la conservation des dents de sagesse mandibulaires}

Le bénéfice lié à la conservation de dents de sagesse mandibulaires est très mal identifié du fait de la qualité et de la quantité des études relativement faibles portant sur le sujet. Deux études en cours depuis 2003 aux Etats-Unis et en Suède ${ }^{[6]}$ n'ont pas permis de comparer les complications des extractions prophylactiques versus l'abstention thérapeutique. Pourtant les complications inhérentes à la conservation des dents de sagesse sont bien identifiées ${ }^{44,28,61]}$ :

- accidents infectieux : péricoronarites aiguës suppurées qui se répètent inexorablement de façon de plus en plus sévère [62], accidents muqueux, osseux, cellulaire [63], ganglionnaire, sinusien, vasculaire, infectieux à distance ;

- accidents mécaniques : lésions de la face distale de la deuxième molaire [64], troubles de l'articulé dentaire, lésions muqueuses mécaniques, fragilisation de l'angle mandibulaire ;

- accidents kystiques : kystes marginaux postérieurs, kystes dentigères [65] ;

- troubles neurologiques, musculaires, algo-dysfonctionnels de l'ATM, trophiques [48].

Les péricoronarites sont les complications les plus fréquentes. Elles peuvent se compliquer de cellulites aiguës qui évoluent dans la région du carrefour oropharyngé et peuvent être une menace grave pour la liberté des voies aériennes.

Esposito [66] indique que certaines données, ne provenant pas d'études randomisées et contrôlées, suggèrent que l'extraction de dents de sagesse asymptomatiques peut être bénéfique en cas de présence d'une carie ou d'une poche parodontale distale à la seconde molaire.

Lee et Dodson [67] affirment que la présence d'une dent de sagesse double le risque de fracture de la mandibule.

\section{Les bénéfices liés à l'avulsion précoce des dents de sagesse mandibulaires}

Concernant les bénéfices liés à l'extraction de dents de sagesse, il semble néanmoins qu'un consensus se dégage sur un point. D'après de nombreux auteurs le bénéfice maximal de l'ex- traction des dents de sagesse concerne les patients jeunes du fait notamment de suites opératoires plus favorables $[4,7,68]$. Le risque de complication des extractions est évalué par Crosse [19] à $12 \%$ chez les 12-29 ans et à $22 \%$ chez les 25-81 ans. C'est pourquoi Mercier et Precious [69] de même que Tate [70] et Brokaw ${ }^{[71]}$ recommandent d'extraire précocement les dents de sagesse. Cette assertion est reprise par I'American Association of Oral and Maxillofacial Surgeons (AAOMS) ${ }^{[72]}$ qui rappelle que plus on attend, plus les dents de sagesse sont difficiles à extraire. L'AAOMS estime que $85 \%$ des dents de sagesse auront éventuellement besoin d'être extraites et préconise fortement une extraction précoce des dents de sagesse, ce qui permet de minimiser les complications. Cette extraction s'impose d'autant plus que le patient est susceptible de se rendre dans une région où il n'y a pas d'accès aisé aux soins dentaires [62].

De plus, certaines lésions, notamment parodontales, peuvent devenir irréversibles si la dent de sagesse n'est pas extraite avant 30 ans [73].

Notons également l'article de Silvertri et Singh [2] qui souligne que les troisièmes molaires présentent une faible valeur fonctionnelle et un taux relativement élevé de douleurs et de pathologies associées. De plus ces dents sont souvent difficiles à traiter de part leur position très postérieure et souvent ectopique (accès limité des instruments rotatifs). De fait leur valeur au sein de la denture est « incertaine et douteuse ».

\section{Limites des publications}

La revue de la littérature montre des résultats disparates quant aux indications ayant conduit à l'extraction des dents de sagesse. Ainsi, la péricoronarite est décrite comme le motif d'extraction des dents de sagesse dans 8 à $59 \%$ des cas selon les auteurs ${ }^{[74-76]}$. De même, la douleur est le motif d'extraction de dents de sagesse pour 5 à $53 \%$ des patients selon une autre étude [77]. Quant à la prévalence de la résorption radiculaire de la deuxième molaire, elle varie de 0 à $24 \%$ selon les auteurs, et de 0 à $11 \%$ pour les kystes dentigères [17]. Le problème est que cette disparité ne permet pas de se forger une opinion déci- médecine

buccale

chirurgie

buccale

VOL. $14, \mathrm{~N}^{\circ} 4$ 2008

page 197 
médecine

buccale

chirurgie

buccale

VOL. $14, \mathrm{~N}^{\circ} 4$ 2008

page 198 sionnelle. En effet, la prise de décision thérapeutique nécessiterait de pouvoir quantifier le bénéfice de l'extraction de dents de sagesse en déterminant scientifiquement la prévalence des complications liées à leur conservation. Mais peut-on quantifier ce risque de complications ? Certains auteurs ne le pensent pas [78-81]. L'Agence Nationale d'Accréditation et d'Evaluation de Santé (ANAES) indique que tant qu'il n'y aura pas un consensus à propos des critères de diagnostic, il ne sera pas possible de déterminer une prévalence ni de comparer les résultats de différentes études [17].

Mais cette absence d'évaluation scientifique du bénéfice lié à la conservation de certaines dents de sagesse permet-elle de conclure comme Jacquemart [82] qu'un tiers des dents de sagesse sont retirées sans raison scientifique prouvée en arguant qu'il n'y a pas d'étude scientifique tendant à dire qu'il faille les conserver? Et qu'en estil de la phrase de Worall [83] qui indique dans un article que des « preuves partielles suggèrent que les dangers de l'avulsion prophylactique des dents de sagesse l'emportent sur ses avantages ». C'est d'ailleurs pourquoi Worall précise qu' « il est vraisemblable qu'une réponse définitive à la question posée (doit-on procéder à l'avulsion prophylactique des dents de sagesse ?) demanderait une étude randomisée et contrôlée avec 10 ans de suivi. » Elle reste à faire.

Et que peut-on conclure cliniquement d'une étude de Brickley et coll. [84] qui affirme que si la probabilité d'une péricoronarite récidivante est supérieure à $52 \%$ alors la décision d'avulsion est préférable à la conservation ?

II faut également être prudent quant à certaines publications présentant des biais ou du moins orientées du fait de la prise en compte de facteurs médicaux mais aussi de critères économiques. Le coût lié aux interventions d'avulsion de dents de sagesse comprend les dépenses d'assurance maladie (largement majorées en cas d'anesthésie générale), le coût des arrêts de travail ${ }^{[26,85]}$ (de 1 à 6 jours selon le cas clinique et le mode opératoire $[31,86,87]$ ) et la prise en charge des suites opératoires [88] (douleurs, trismus, œdème, atteintes nerveuses temporaires ou définitives). La dépense occasionnée par l'avulsion d'une troi- sième molaire mandibulaire asymptomatique serait de $33 \%$ plus élevée que celle liée à l'abstention thérapeutique [89]. On comprend la tentation économique d'une approche conservatrice des dents de sagesse. Le coût des extractions de dents de sagesse en Angleterre et au Pays de Galles est estimé à 12 millions de livres sterling annuels et 5 millions pourraient être économisés en modifiant les pratiques des chirurgiens den-

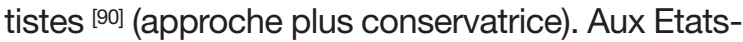
Unis, le montant dépensé chaque année pour l'extraction des dents de sagesse est estimé à plus de 2 milliards de dollars (sans prendre en compte le coût des examens, des radiographies, de l'anesthésie, de l'hospitalisation) [91]. En France, l'extraction des dents de sagesse représente, selon un rapport de la CPAM de 1992, 45,1\% des coefficients se rapportant à l'extraction de dents permanentes [92].

Au total l'évaluation du rapport bénéfices/risques de l'extraction de dents de sagesse asymptomatiques est délicate. Dans le cadre d'une revue systématique d'études contrôlées randomisées effectuées au profit du Cochrane collaboration [93], les auteurs n'ont trouvé aucune preuve permettant de soutenir ou au contraire de réfuter la pratique consistant à extraire des dents de sagesse asymptomatiques.

\section{Vers un consensus français ?}

En France, le texte de référence en matière de prise en charge des dents de sagesse a été publié par l'ANAES [17] en 1997 sous le titre « indications et non-indications de l'avulsion des troisièmes molaires mandibulaires. Recommandations et références médicales ". A la lecture de cette publication, il apparaît tentant de n'en retenir qu'une seule phrase : «l'analyse de la littérature n'a pas permis d'identifier des articles pour établir des recommandations professionnelles incontestables ". II est cependant indispensable de comprendre ce qui a amené les auteurs de cette étude à cette assertion.

II faut tout d'abord se rendre compte que l'ANAES a sélectionné et analysé pour son étude 112 articles dont 61 pour l'élaboration du texte de recommandations. Mais l'ANAES s'est très vite 
retrouvée confrontée à des différences dans la terminologie utilisée par les auteurs qui ont limité la valeur des résultats des études épidémiologiques. II apparaît ainsi tout de suite que la question récurrente qui se pose aux chirurgiens dentistes civils comme militaires « faut-il extraire les dents de sagesse? » n'a aucun sens. Jusqu'à preuve du contraire, la dent de sagesse n'est pas une pathologie. C'est pourquoi il faut s'attacher dans un premier temps à la terminologie des cas auxquels on est confronté. Comme cela a été fait par l'ANAES.

Les termes employés sont définis ainsi :

- un germe dentaire de dent permanente : cet état correspond au stade primitif d'une dent, depuis le stade de bourgeon jusqu'à la maturation complète de la dent, c'est-à-dire jusqu'à ce que l'édification radiculaire soit achevée ;

- une dent permanente incluse : il s'agit d'une dent mature qui n'a pas fait son éruption après la date physiologique et dont le sac péricoronaire ne présente pas de communication avec la cavité buccale. Une dent incluse est recouverte ou non par du tissu osseux mais elle l'est totalement par la muqueuse buccale ;

- une dent retenue : c'est une dent immature gênée dans son éruption et qui conserve un potentiel évolutif. L'édification radiculaire n'est pas terminée (apex ouvert). Avec la maturation de la dent, la rétention évolue vers l'inclusion ou l'enclavement ; - une dent en désinclusion : lorsqu'étant préalablement incluse, elle devient exposée au milieu buccal ;

- une dent enclavée : c'est une dent mature, incluse ou non, dont l'éruption s'arrête du fait d'un obstacle. L'obstacle étant éliminé, cette dent qui conserve un potentiel évolutif, peut poursuivre son éruption.

Les dents de sagesse peuvent ainsi être pathologiques (symptomatiques ou non) ou saines (symptomatiques ou non). Les pathologies liées à ces dents de sagesse sont portées par les dents elles-mêmes (caries...) ou non (atteintes parodontales, kystes dentigères...), elles sont multiples et nécessitent des décisions thérapeutiques spécifiques.

Dire qu'il n'y a pas de consensus quant à l'extraction des dents de sagesse n'est pas tout à fait exact. II apparaît clairement à la lecture des recommandations de l'ANAES :

- qu'il est recommandé de conserver les dents profondément incluses et asymptomatiques qui ont une faible probabilité de faire éruption ou d'être à l'origine de pathologies. Le patient doit alors être informé de la nécessité d'un suivi clinique et radiologique régulier. Quoi qu'il en soit, il faut bien faire comprendre au patient la nécessité d'avulsion en cas d'évolution radiologique ou clinique défavorable, même en l'absence de symptôme.

- que les dents de sagesse présentant une pathologie non traitable par des soins conservateurs simples (dent délabrée, à l'origine de péricoronarites récidivantes...) doivent être extraites.

Il est important de noter que les dents de sagesse à l'origine de plusieurs épisodes de péricoronarite ou d'une complication kystique doivent être extraites dans le respect des recommandations de l'ANAES.

Par contre, à la différence des cas précédemment cités, il existe un domaine où le consensus est totalement absent : l'avulsion prophylactique des dents de sagesse. L'indication d'avulsion prophylactique se fonde essentiellement sur la supposition qu'une complication interviendra de manière inévitable, à un moment ou un autre, au cours de la vie de tout individu. L'intérêt de l'avulsion prophylactique des troisièmes molaires mandibulaires est de réduire le risque de développer des affections telles que les infections péricoronaires, les lésions de la dent adjacente, les kystes dentigères, les tumeurs malignes et probablement des complications chirurgicales plus sévères si l'avulsion est pratiquée à un âge avancé. L'ANAES affirme que les avulsions précoces sont encouragées par des observations montrant que la morbidité est moindre chez le sujet jeune. Le problème est que la justification de ces avulsions prophylactiques de troisièmes molaires incluses et asymptomatiques repose sur des opinions, des cas ou des séries de cas cliniques, des études ayant des biais méthodologiques (échantillons sélectionnés non représentatifs de la population générale).

Mais il ne faut pas confondre extraction prophylactique et extraction de dents asymptomatiques, voire saines. L'ANAES rappelle que l'extraction médecine

buccale chirurgie buccale

VOL. $14, \mathrm{~N}^{\circ} 4$ 2008

page 199 
d'une dent en malposition n'est pas un traitement considéré comme prophylactique et suggère l'avulsion des dents de sagesse en désinclusion, même en l'absence de symptôme. L'ANAES indique ainsi que les dents partiellement recouvertes de tissus mous sont à l'origine de pathologies (essentiellement de péricoronarites). Ces recommandations, soutenues par des faits scientifiques ${ }^{[94]}$ et confirmées par l'expérience clinique, trouvent leur application en pratique clinique puisque ces avulsions représentaient de 18 à $50 \%$ des avulsions de dents de sagesse mandibulaires en 1993 en Angleterre [95-97] et 70,3 \% dans les années 1980 en Suède [98].

\section{Vers un consensus international ?}

Les plus grands organismes médicaux internationaux se sont penchés sur la question de l'ex-

médecine

buccale

chirurgie

buccale

VOL. $14, \mathrm{~N}^{\circ} 4$ 2008

page 200 traction des dents de sagesse mais beaucoup n'ont fait que souligner les controverses [11]. Le NIHCE (National Institute for Health and Clinical Evidence) a ainsi publié un guide sur l'avulsion des dents de sagesse en $1980{ }^{[99]}$ et un deuxième, toujours en vigueur, en $2000{ }^{[6]}$. Dans ce guide, le NIHCE affirme que l'extraction prophylactique des dents de sagesse incluses saines doit être stoppée. Ceci est intéressant puisqu'à la notion d'inclusion le NIHCE adjoint celle de saine et permet ainsi de bien comprendre qu'il faut toujours juxtaposer la position (incluse...) et l'état de la dent (saine...) pour prendre une décision thérapeutique. Notons que la terminologie anglo-saxonne ne correspond pas à celle employée par l'ANAES puisque le terme « impacted » définit aussi bien une dent qui n'a pas fait son éruption complète mais en position fonctionnelle normale, du fait d'un manque de place sur l'arcade dentaire, d'une obstruction par une autre dent ou de son développement en position anormale [100, 101]. La même dénomination s'applique donc à des dents complètement ou partiellement incluses. II est de fait difficile de trouver un consensus lorsque les constations initiales ne sont pas posées de la même façon entre les pays. Ainsi pour l'extraction prophylactique des dents de sagesse, le NIHCE indique qu'il n'y a pas de preuve fiable montrant le bénéfice des extractions prophylac- tiques des dents de sagesse « impacted », ce qui est en accord avec la littérature française si l'on ne considère que les dents incluses mais ne l'est plus si l'on considère les dents en désinclusion. Par contre, le NIHCE reprend certaines recommandations de l'ANAES :

- les patients qui ont une dent de sagesse incluse qui ne pose pas de problème doivent la conserver et la faire contrôler régulièrement chez leur chirurgien dentiste ;

- les patients qui ont une pathologie liée à leur dent de sagesse doivent la faire extraire (carie intraitable, abcès, kyste, tumeur, pathologie parodontale, présence de la dent dans la voie d'abord d'une autre intervention chirurgicale).

Une phrase de ce guide clinique semble très importante à retenir : " l'impaction est une anormalité qui prédispose à des évolutions pathologiques telles que les péricoronarites, caries, résorptions et problèmes parodontaux. Des kystes et des tumeurs peuvent aussi se développer. " Le NIDR (National Institute of Dental Research), dans sa conférence de consensus sur l'extraction des troisièmes molaires [7], reprend les écrits de l'ANAES en affirmant que " si la désinclusion n'est pas pathologique en elle-même, le consensus du NIDR considère que la désinclusion ou la malposition des dents de sagesse est un état anormal qui peut justifier leur extraction : de tels traitements ne sont pas considérés comme prophylactiques ».

Deux situations sont présentées comme ayant un fort potentiel de conséquences pathologiques locales:

- quand une dent de sagesse impactée est verticale ou disto-versée, proche ou au niveau du plan d'occlusion mais recouverte sur la moitié de sa surface ou plus par des tissus mous, la probabilité de péricoronarite augmente fortement ${ }^{[94,102]}$; - quand une dent de sagesse impactée est en position mésio-angulaire ou horizontale, avec un contact direct ou proche du contact avec la jonction amélo-cémentaire de la seconde molaire, le risque de carie de la deuxième molaire augmente particulièrement lorsque l'hygiène bucco-dentaire du sujet est limitée.

Cette dernière situation est intéressante car les auteurs associent le terrain à la disposition anato- 
mique de l'organe dentaire faisant ainsi mention de facteurs extrinsèques qui peuvent prendre une place prépondérante dans la décision d'extraction. Le NHS quant à lui indique qu'une avulsion prophylactique est préconisée pour prévenir d'éventuelles complications, notamment pour les dents partiellement évoluées sans probabilité d'évolution complète [9].

\section{Conséquences en pratique quoti- dienne civile}

L'absence totale de consensus dans le cadre de l'extraction de certaines dents de sagesse conduit à une situation qui peut paraître ubuesque : Knutsson et coll., dans une étude portant sur l'indication d'extraction de troisièmes molaires mandibulaires asymptomatiques [103], ont demandé à 30 chirurgiens dentistes de donner leur avis sur 36 dents de sagesse. Pour ces praticiens, le nombre de molaires à extraire varie de 0 à 26 et il n'y a qu'un cas pour lequel les praticiens proposaient l'extraction comme unique attitude thérapeutique.

Une deuxième étude a été menée par Jones et coll. [104] auprès de 74 chirurgiens dentistes. Les auteurs ont soumis aux praticiens 23 cas cliniques avec anamnèse, photos et radiographies. Pour les praticiens, l'extraction s'impose dans une fourchette allant de 0 à 21 cas.

Il est facile à comprendre que certains praticiens conseillent à leurs patients l'avulsion de troisièmes molaires mandibulaires asymptomatiques alors que d'autres recommandent une attitude conservatrice en établissant une surveillance régulière et en attendant éventuellement le développement d'une pathologie pour intervenir [105]. Cette variation dans l'attitude pratique d'un chirurgien dentiste à l'autre s'explique selon Hazelkorn et Macek [106] par des habitudes, des croyances, le type de formation et le mode de rémunération des praticiens. Lopes et coll. parlent même de préjugés des praticiens [96], ce que soulignent également Brickley et coll. [108]. Ceci amène l'ensemble de ces auteurs à affirmer que les décisions médicales ne reposent pas assez sur des faits, sur des réalités cliniques et sur des preuves. Un exemple est prégnant dans le cadre de la prise de décision d'extraction par les chirurgiens dentistes : certains praticiens pensent que l'éruption des troisièmes molaires mandibulaires serait à l'origine du chevauchement des dents mandibulaires. L'implication des dents de sagesse dans l'encombrement dentaire est discutée depuis des décennies et aucune étude n'a apporté de preuve irréfutable, pourtant de nombreuses dents de sagesse sont encore extraites pour ce motif [108-111].

Par contre, si l'attitude pratique du corps médical varie beaucoup, il n'en va pas de même des patients qui, conformément à ce que l'on peut penser, penchent très largement en faveur de la conservation de leurs dents de sagesse [112].

Au total, il semble que la diffusion des connaissances scientifiques actuelles aboutisse à un consensus portant sur :

- l'extraction des dents de sagesse pathologiques non traitables par des soins conservateurs ou en présence de pathologies associées aux dents de sagesse ;

- la conservation des dents de sagesse saines profondément incluses en l'absence de pathologie associée à la dent.

II ne reste alors que deux situations problématiques qui sont malheureusement les plus fréquentes:

- l'extraction des dents de sagesse après un épisode de péricoronarite (la récurrence des épisodes impliquant l'extraction n'est pas définie) ; - et surtout l'extraction des dents saines et asymptomatiques en désinclusion.

\section{APPROCHE MILITAIRE : PRISE EN COMPTE DES PARTICULARITES INHERENTES A L'ACTIVITE OPERATIONNELLE DES MILITAIRES}

\section{Approche militaire internationale}

Dans le cadre de la prise en charge des dents de sagesse, chaque praticien militaire français ou étranger, en plus de la connaissance des recommandations de l'ANAES, du NHS... doit garder à l'esprit les spécificités du métier de militaire. Ces particularités inhérentes à la vie de militaire trou- médecine

buccale

chirurgie

buccale

VOL. $14, \mathrm{~N}^{\circ} 4$ 2008

page 201 
médecine

buccale

chirurgie

buccale

VOL. $14, \mathrm{~N}^{\circ} 4$ 2008

page 202 vent leur quintessence dans les opérations extérieures qui vont avoir un impact considérable sur l'environnement des personnels et sur leur santé bucco-dentaire.

L'objectif des mises en condition dentaire avant projection est d'amener l'ensemble des personnels à un état bucco-dentaire limitant les consultations durant les opérations extérieures aux seuls motifs imprévisibles (traumatisme, fractures dentaires...). C'est à cette fin qu'a été mis en place le STANAG 2466 [113], texte de standardisation de I'OTAN visant à identifier les personnels dont l'état bucco-dentaire risque d'être à l'origine d'une consultation dentaire en urgence sur une période de 12 mois. II est démontré que l'impact des pathologies bucco-dentaires sur la capacité opérationnelle d'une Unité peut être délétère car tout personnel devant consulter un chirurgien dentiste sur un théâtre d'opération extérieure doit nécessairement quitter son poste, être accompagné par d'autres soldats (plus ou moins nombreux en fonction de la dangerosité de la mission) et ce à bord de véhicules (terrestres, aériens...). Ceci représente une diminution de la disponibilité opérationnelle de personnels, de matériels pouvant aller jusqu'à hypothéquer une mission. Au-delà de la mission, ces transports de personnels sur tous les théâtres d'opération représentent un coût (transport) mais aussi un risque certain [114-116] (accidents de transport, attaques et engins explosifs imprévus...).

Dès lors une question se pose : les urgences dentaires liées aux dents de sagesse font-elles partie des complications prévisibles ou non? Du fait de son fort taux de projection, l'armée américaine s'est largement penchée sur la question [117]. II en ressort que les conditions de vie sur le terrain ont un impact tel sur le militaire (hygiène alimentaire, hygiène bucco-dentaire, stress...) que certaines dents de sagesse qui n'auraient pas été extraites si le personnel était resté sur le sol américain devenaient source d'inaptitude du fait des complications qu'elles pouvaient générer au cours d'une mission. «Beaucoup de militaires cessent de se brosser les dents quotidiennement durant les projections, ajouter à cela des habitudes nocives comme la consommation accrue de tabac et de boissons sucrées ainsi que le snac- king notamment pour lutter contre l'ennui ou le stress, ceci peut clairement être à l'origine de problèmes de dents de sagesse notamment sur les théâtres d'opérations extérieures » [17]. L'armée anglaise a également durci ses critères d'aptitude concernant les dents de sagesse en concluant que la fréquence élevée des péricoronarites durant les projections est liée à une diminution des défenses immunitaires des individus consécutive au stress, aux modifications du comportement de vie, au changement des habitudes alimentaires... Ces péricoronarites devenaient de fait prévisibles. Cette assertion est confirmée par l'armée américaine ${ }^{[118,119]}$ et l'armée australienne [120]. De plus la réalisation d'extractions de dents de sagesse sur les théâtres d'opérations extérieures peut s'avérer délicate et chronophage pour le personnel (traiter l'infection, intervenir, attendre la cicatrisation...) avec des résultats aléatoires surtout en terme de cicatrisation du fait des conditions de vie sur le terrain.

En ce qui concerne l'aptitude dentaire, le STANAG 2466 prévoit 4 classes d'aptitude en fonction de l'état bucco-dentaire des militaires [121, 122] : - classe 1 : absence de pathologie ;

- classe 2 : présence de pathologies sans risque de survenue d'urgences dentaires sur une période de 12 mois ;

- classe 3 : présence de pathologies avec risque de survenue d'urgences dentaires sur une période de 12 mois ;

- classe 4 : statut dentaire inconnu (personnel non examiné, absence de radiographie panoramique dentaire).

La prise en compte des dents de sagesse dans le STANAG 2466 est la suivante :

- sont classés 2 : les personnels ayant une dent de sagesse incluse, en désinclusion ou en malposition sans signe clinique ou radiologique, sans antécédent de pathologie ou sans symptôme pathologique ;

- sont classés 3 : les personnels ayant une dent de sagesse en désinclusion ou en malposition avec signes cliniques ou radiologiques, des antécédents de pathologies ou des symptômes pathologiques (un seul critère suffit pour être classé 3). Ces dents doivent être extraites dans le cadre du rétablissement de l'aptitude. 
Au fil des années et des retours d'expérience de missions extérieures, certaines dents de sagesse, notamment celles en désinclusion avec un seul antécédent de péricoronarite, ont progressivement glissé de la classe 2 à la classe 3 [123].

Le revirement de position le plus net concernant la prise en charge des dents de sagesse mandibulaires a été celui de l'armée australienne. Dans le passé, les retours d'expérience publiés par l'armée australienne ${ }^{[119]}$ indiquaient que les péricoronarites étaient un problème majeur et le motif de nombreuses évacuations sanitaires. La prévalence des urgences liées aux dents de sagesse au sein de l'armée australienne avait pour étiologie, outre les conditions de vie sur le terrain, la population militaire elle-même : une population jeune, dans la tranche d'âge la plus sensible aux péricoronarites ${ }^{[124]}$. De ce fait, le service de santé des armées australien a modifié sa politique de prise en charge des dents de sagesse [125]. L'extraction des troisièmes molaires mandibulaires en désinclusion a conduit à une diminution significative de ce type de problème. Durant l'opération Warden (Timor Oriental), il était estimé, sur la base d'études épidémiologiques antérieures, que les forces projetées connaîtraient 90 à 100 cas de péricoronarites. Dans les faits il n'y eu que 8 cas. Ceci se traduit par une préservation de la capacité opérationnelle des militaires avec moins de jours d'indisponibilité et moins d'évacuations sanitaires ${ }^{[126] .}$

En fait, les problèmes rencontrés par une population civile dans des conditions de vie normale [87] sont exacerbés au sein de la population militaire au cours des projections. Cette constatation a été faite au sein de toutes les armées dont le protocole de mise en condition dentaire a été étudié.

\section{Approche militaire française}

En ce qui concerne l'Armée française, deux notes de la Direction Centrale du Service de Santé des Armées font autorité pour la prise en charge des dents de sagesse dans le cadre de l'aptitude Opex outre-mer. La première note datée de 1994 [127] indique que les dents de sagesse profondément incluses ou celles qui peuvent trouver, après évolution, une place sur l'arcade dentaire, doivent être respectées et ne pas entraîner d'inaptitude. Une attitude inverse s'impose en cas de péricoronarite ou devant une dent de sagesse en désinclusion et qui ne peut pas trouver une place sur l'arcade. La deuxième note datée de $1995{ }^{\left[{ }^{128]} \text { pré- }\right.}$ cise que les avulsions de dents de sagesse ne doivent en aucun cas être réalisées systématiquement sur simple motif « d'aptitude outre-mer» mais toujours décidées au cours d'une démarche thérapeutique adaptée à la pathologie dépistée. L'approche curative des soins est ainsi rappelée.

\section{DISCUSSION}

La pratique de la chirurgie dentaire en milieu militaire ne peut et ne doit en aucun cas exonérer une approche thérapeutique fondée sur la preuve et les faits. On doit donc se conformer aux recommandations de bonnes pratiques en vigueur dans le cadre de la prise de décision d'extraire ou non certaines dents de sagesse mandibulaires à savoir :

- extraction des dents de sagesse pathologiques ou en présence de pathologies liées aux dents de sagesse (caries « intraitables », antécédents de péricoronarite, kystes marginaux...) ;

- conservation des dents de sagesse saines profondément incluses en l'absence de pathologie associée à la dent (à surveiller).

En ce qui concerne les situations cliniques ne faisant pas l'objet de consensus, il semble tout d'abord important de bannir de notre vocabulaire l'expression « extraction prophylactique » lorsqu'il s'agit des dents en désinclusion même lorsqu'elles sont saines. II ne faut en aucun cas laisser croire au caractère systématique des extractions dentaires du seul fait de l'aptitude à la projection. Ces extractions n'auraient aucune justification, ni réglementaire (notes de la DCSSA), ni scientifique (ANAES, publications internationales).

Face aux dents de sagesse mandibulaires en désinclusion, la nécessité de la prise de décision d'extraire ou non doit amener à une démarche identique à celle des praticiens civils en posant la question : les bénéfices apportés par l'intervention sont-il supérieurs pour le patient aux médecine

buccale chirurgie buccale

VOL. $14, \mathrm{~N}^{\circ} 4$ 2008

page 203 
médecine

buccale

chirurgie

buccale

VOL. $14, \mathrm{~N}^{\circ} 4$ 2008

page 204 risques liés à l'extraction ? Par contre, les éléments permettant de répondre à cette question diffèrent dans le milieu militaire par rapport au civil. Et surtout, il y a un déplacement du curseur bénéfices/risques du coté bénéfices car :

- L'opération extérieure est le lieu où le risque de survenue d'urgences dentaires devrait être minoré au maximum de façon à maintenir la capacité opérationnelle de l'Unité : or il s'avère que les théâtres d'opération sont un lieu de majoration maximale de ce risque car la modification de l'hygiène bucco-dentaire et alimentaire, la fatigue, le stress mais aussi l'accès limité aux soins dentaires sur certains théâtres d'opération constituent des facteurs péjoratifs pour la sphère bucco-dentaire en général et pour les dents de sagesse en particulier. L'extraction précoce de certaines dents de sagesse, notamment celles en désinclusion, apporte donc un bénéfice particulier aux personnels militaires en les préservant de déconvenues majeures durant leur projection. C'est d'ailleurs la totalité de l'Unité qui bénéficie de ces extractions de dents de sagesse en terme de disponibilité et de maintien de sa capacité opérationnelle.

- L'extraction des dents de sagesse chez des sujets jeunes, comme c'est le cas au sein de la population militaire, permet de minorer le risque de survenue de complications post-opératoires. Il est important de préciser que si le curseur bénéfices/risques est déplacé dans le cas de militaires projetés, il n'est en aucun cas bloqué sur « bénéfices ». Les situations anatomiques délicates, notamment du fait de la proximité du nerf mandibulaire, doivent être soigneusement identifiées et peuvent justifier, comme dans le civil, l'abstention thérapeutique.

Au total, dans le souci du maintien de la capacité opérationnelle des Forces armées, il apparaît indispensable d'appliquer de façon claire et systématique la note $\mathrm{N}^{\circ}$ 988/DEF/DCSSA/AST/TEC/1 du 21 avril 1994 relative à l'aptitude « dentaire » à servir Outre-mer ou en opération extérieure qui impose l'extraction des dents de sagesse présentant des antécédents de péricoronarite ou celles en désinclusion qui ne peuvent trouver leur place sur l'arcade. Ceci doit conduire, dans le souci d'une harmonisation de l'aptitude dentaire OTAN et le respect du STANAG 2466, à classer 3 les personnels qui présentent une ou plusieurs dents de sagesse en désinclusion ainsi que ceux ayant fait l'objet d'une ou plusieurs péricoronarites.

\section{CONCLUSION}

La prise de décision d'extraire ou non une dent de sagesse mandibulaire dans le cadre militaire ne se traduit en aucun cas par une exonération des recommandations émanant du milieu médical civil. Le rapport bénéfices/risques de l'intervention pour le patient doit être clairement posé afin de permettre d'orienter la décision thérapeutique dans le respect de l'odontologie fondée sur la preuve.

Dans le cadre de la détermination de l'aptitude à la projection, il apparaît indispensable d'intégrer dans l'analyse du rapport bénéfices/risques les paramètres inhérents aux conditions de vie des militaires (paramètres non pris en compte par les praticiens civils). Ceci a conduit l'ensemble des services de santé des armées ayant étudié le protocole de mise en condition dentaire, comme le service de santé des armées français, à définir clairement l'attitude à adopter :

- conservation des dents de sagesse saines profondément incluses en l'absence de pathologie associée à la dent ;

- conservation des dents de sagesse saines, sans pathologies associées lorsqu'elles se trouvent en normoposition ;

- extraction des dents de sagesse pathologiques non traitables ou en présence de pathologies liées aux dents de sagesse ;

- extraction des dents présentant des antécédents de péricoronarite ;

- extraction des dents de sagesse en désinclusion qui ne peuvent pas trouver leur place sur l'arcade.

Seule l'application claire et systématique de ces recommandations permettra de maintenir la capacité et la disponibilité opérationnelles des Forces armées. 


\section{RÉFÉRENCES}

1 - Sutherland SE. Evidence Based Dentistry: part I. Getting Started. J Can Dent Assoc 2001 ; 67 : 204-6.

2 - Silvestri AN, Singh I. The unresolved problem of the third molar. Would people be better off without it ? J Am Dent Assoc 2003 ; 134 : 450-455.

3 - Ten Cate AR. Oral histology: development, structure, and function (pp. 95). Mosby, St Louis, 5th ed., 1998.

4 - Peron JM. Accidents d'évolution des dents de sagesse. Encycl Med Chir Stomatologie/Odontologie, 22-032-E-10, 2003.

5 - Zhao Z, Weiss KM, Stock DW. Development and evolution of dentition patterns and their genetic basis (pp. 152-72). Cambridge University Press, New York, 2000.

6 - National Institute for Health and Clinical Excellence. Guidance on the removal of wisdom teeth, technology appraisal guidance $N^{\circ} 1$, march 2000. Review march 2003. http://www.nice.org.uk (accessed on August 2006)

7 - National Institute of dental research. Removal of third molars - conférence de consensus, Bethesda, 1979.

8 -Scottish intercollegiate guidelines network. Management of unerupted and impacted third molar teeth - a national clinical guideline. Edinburgh 2000.

9 - NHS. Prophylactic removal impacted third molars: is it justified? NHS for reviews and dissemination, effectiveness matters. University of York $1998 ; 3: 4$.

10 - Royal College of Surgeons of England. Faculty of Dental Surgery: National Clinical Guidelines, 1997. http://www.rcseng.ac.uk/fds/docs/ncg97.pdf (accessed 25 August 2006).

11 - National institute of dental research (NIH). Removal of third molars. Bethesda: NIDR 1979.

12 - Song F, Landes DP, Glenny AM, Sheldon TA. Prophylactic removal of impacted third molars: an assessment of published reviews. Br Dent J 1997 ; $182: 339-46$.

13 - News. Oldest recorded case of impacted wisdom teeth. Br Dent J $2005 ; 200: 311$.

14 - Anderson BL, Thompson GW, Popovitch F. Evolutionary dental changes. Am J Phys Anthropol $1975 ; 43:$ 95-102.

15 - Nanda RS. Agenesis of third molar in man. Am J Orthodont $1954 ; 40: 698-706$.

16 - Buisson G. Traitement des dents incluses (autres que les dents de sagesse et que les canines supérieures). Encycl Méd Chir - Chirurgie bucco-dentaire 22096 F10, 1968.

17 - Agence nationale d'accréditation et d'évaluation de santé. Indications et non-indications de l'avulsion des troisièmes molaires mandibulaires. Recommandations et références médicales, 1997.

18 - Sands T, Pynn BR, Nenninger S. Third molar surgery: current concepts and controversies, part 2. Oral Health 1993 ; 83 : 19-30.
19 - Crosse A. Wisdom teeth removal. NHS. Reviewed policy - 5 April 2004

20 - Hugoson A, Kugelberg CF. The prevalence of third molars in a Swedish population. An epidemiological study. Community Dent Health 1988 ; 5 : 121-38.

21 - Bjork A, Jensen E, Palling M. Mandibular growth and third molar impaction. Acta Odontol Scand 1956 ; $14: 231-72$.

22 - Stanley HR, Alattar M, Collett WK, Stringfellow HR, Spiegel EH. Pathological sequelae of "neglected" impacted third molars. J Oral Pathol 1988 ; 17 : 113-7.

23 - Shepherd JP, Brickley M. Surgical removal of third molars. Br Med J 1994 ; 309 : 620-621.

24 - Sands T, Pynn BR, Nenniger S. Third molar surgery: current concepts and controversies, part 1. Oral Health $1993 ; 83: 11-4,17$.

25 - Anastasio D, Giraud E, Osswald JM. La chirurgie buccale ambulatoire : étude rétrospective sur l'année 2001. Med Buccale Chir Buccale 2002 ; 8 : 22-6.

26 - Fouilloux I, Torres JH, Pasquet G, Cavezian R, Chambas C. De l'utilité de la dent de sagesse (pp. 315-9). Quintessence ADF, 2006.

27 - Pilot Clinical Guidelines. British Association of Oral \& Maxillofacial Surgeons. London, 1995.

28 - Jacquemart P, Dart T. Conserver ou extraire les dents de sagesse. Info Dent 2005 ; 25 : 1489-95.

29 - Tulloch JFC, Antczak AA, Wilkes JW. The application of decision analysis to evaluate the need for extraction of asymptomatic third molars. J Oral Maxillofac Surg $1987 ; 45: 855-63$.

30 - Harradine NW, Pearson MH, Toth B. The effect of extraction of third molars on late lower incisor crowding: a randomized controlled trial. $\mathrm{Br} \mathrm{J}$ Orthodont $1998 ; 25: 117-22$.

31 - Société française de stomatologie et chirurgie maxillofaciale. Information médicale avant l'extraction des dents de sagesse. Rev Stomatol Chir Maxillofac 2008 ; 109 : 67-68.

32 - Bramley P. Sense about wisdom ? J Royal Soc Med $1981 ; 74: 867-9$.

33 - Moghadam HG, Caminiti MF. Life-threatening haemorrhage after extraction of third molars: case report and management protocol. J Can Dent Assoc 2002 ; $68: 670-4$

34 - Sisk AL, Hammer WB, Shelton DW, Joy ED Jr. Complications following removal of impacted third molars: the role of the experience of the surgeon. $J$ Oral Maxfac Surg 1986 ; 44 : 855-9.

35 - Torres JH. Bénéfice et risque des avulsions de dents de sagesse. Rev Stomatol Chir Maxillofac 1997 ; 98 : 277-80

36 - Bell GW. Use of dental panoramic tomographs to predict the relation between mandibular third molar teeth and the inferior alveolar nerve. Radiological and sur- \begin{tabular}{l}
$\begin{array}{l}\text { médecine } \\
\text { buccale } \\
\text { chirurgie } \\
\text { buccale }\end{array}$ \\
\hline VOL. $14, N^{\circ} 4$ \\
2008 \\
\hline page 205
\end{tabular} 
gical findings, and clinical outcome. $\mathrm{Br} J$ Oral Maxillofac Surg 2004 ; 42 : 21-7.

37 - Daneluzzo C. Germectomie avec imagerie par reconstruction informatique 3D. Chir Dent Fr 2004 ; 1184 : 59-66.

38 - Scottish intercollegiate network. Management of unerepted and impacted third molar teeth. March 2000. SIGN Publication n43. http://www.sign.ac.uk (accessed on 25 August 2006).

39 - Weil K, Hooper L, Afzal Z, Esposito M, Worthington HV, van Wijk AJ, Coulthard P. Paracetamol for pain relief after surgical removal of lower wisdom teeth (protocol) (Cochrane review). In: the Cochrane Library, 2006, Issue 2. Wiley, Chichester, UK.

40 - Chi CH, Seldin EB, Dodson TB. Types, frequencies, and risk factors for complications after third molar extraction. J Oral Maxillofac Surg 2003 ; 61 : 137989.

41 - Figueiredo R, Valmaseda-Castellom E, Berini-Aytes L, Gay-Escoda C. Incidence and clinical features of delayed-onset infections after extraction of lower third molars. Oral Surg Oral Med Oral Pathol Oral Radiol Endod 2005 ; 99 : 265-9.

buccale

chirurgie

buccale

VOL. $14, \mathrm{~N}^{\circ} 4$ 2008

page 206
42 - Haug RH, Perrot DH, Gonzales MJ, Talwar RM. The American Association of Oral and Maxillofacial Surgeons age-related third molar study. J Oral Maxillofac Surg 2005 ; 63 : 1106-14.

43 - Larsen PE. Alveolar osteitis after surgical removal of impacted mandibular third molars: identification of the patient at risk. Oral Surg Oral Med Oral Pathol 1992 ; $73:$ 393-7.

44 - Academy of general dentistry. Check menstrual calendar for tooth extraction. http://www.agd.org/consumer/topics/wisdom/drysocket.asp.

45 - Kugelberg CF, Ahlstrom U, Ericson S, Hugoson A. Periodontal healing after impacted lower third surgery: a retrospective study. Int J Oral Surg 1985 ; 14 : 29-40.

46 - Kugelberg CF. Periodontal healing of two and four years after impacted lower third molar surgery: a comparative retrospective study. Int J Oral Maxillofac Surg $1990 ; 19: 341-5$.

47 - Valmasseda-Castellan E, Berini-Aytes L, GayEscoda C. Inferior alveolar nerve damage after lower third molar surgical extraction: a prospective study of 1,117 surgical extractions. Oral Surg Oral Med Oral Pathol Oral Radiol Endod 2001 ; 92 : 377-83.

48 - Taisse S, Shajri S, Riahi A, Benyahya I. Pelade et évolution d'une dent de sagesse mandibulaire : présentation d'un cas clinique. Med Buccale Chir Buccale $2005 ; 11: 81-8$.

49 - O’Riordan B. Coronectomy. Oral Surg Oral Med Oral Pathol $2001 ; 98: 471-88$

50 - Blackburn CW, Bramley PA. Lingual nerve damage associated with the removal of lower third molars. $\mathrm{Br}$ Dent J 1989 ; 167 : 103-7.

51 - Schultz-Mosgau S, Reich RH. Assessment of inferior alveolar and lingual nerve disturbances after dentoal- veolar surgery, and recovery of sensitivity. Inter J Oral Maxillofac Surg $1993 ; 22: 214-7$.

52 - Queral-Godoy E, Valmaseda-Castellon E, BeriniAytes L, Gay-Escoda C. Frequency and evolution of lingual nerve lesions following lower third extraction. J Oral Maxillofac Surg 2006 ; 64 : 402-7.

53 - Rood JP. Permanent damage of inferior alveolar and lingual nerves during the removal of impacted mandibular third molars: comparison of two methods of bone removal. Br Dent J 1992 ; 172 : 108-10.

54 - Commissionat Y. Prévention des lésions neurologiques post-extractionnelles par coronectomie de la dent de sagesse inférieure ( $1^{\text {re }}$ partie). Info Dent $2005 ; 33$ : 1989-92.

55 - Rood J, Sheehab B. The radiological prediction of inferior alveolar nerve injury during third molar surgery. Brit J Oral Maxillofac Surg 1990 ; 28 : 20-5.

56 - Commissionat Y. Prévention des lésions neurologiques post-extractionnelles par coronectomie de la dent de sagesse inférieure ( $2^{e}$ partie). Info Dent 2005 ; 34 : 2053-8.

57 - Robinson PP, Smith KG. Lingual nerve damage during lower third molar removal: a comparison of two surgical methods. Br Dent J 1996 ; 180 : 456-61.

58 - Barda L. Apex des dents de sagesse et canal mandibulaire. Lésions neurologiques et leurs traitements, attitude opératoire. Article mis en ligne sur Dental espace.com le 6 février 2002. http://www.dentalespace.com/dentiste/formation/135-apex-dentssagesse-canal-mandibulaire-lesions-neurologiquesleurs-traitements-attitude-operatoire.htm.

59 - Andreasen JO, Hakansson L. Atlas de réimplantation et de transplantation dentaires. Masson, Paris, 1994.

60 - Peters H, Balling R. Teeth: where and how to make them. Trends Genet $1999 ; 15: 59-65$.

61 - Mourshed F. A roentgenographic study in detecting dentigerous cysts in the early stages. Oral Surg 1964 ; $18: 47-53$.

62 - Carnegie dental group. To keep or not to keep: wisdom teeth. www.carnegiedentalgroup.com. au/PDF/Wisdon_Teeth.pdf.

63 - Rhuin B, Bertrand JC. Abcès et cellulites d'origine dentaire. Le pronostic vital peut être mis en jeu. Concours Med 2005 ; 127 : 1473-77.

64 - Song F, O'Meara S, Wilson P, Golder S, Kleijnen J. The effectiveness and cost-effectiveness of prophylactic removal of wisdom teeth. Health Technology Assesment $2000 ; 4: 1$ :55.

65 - Shepherd JP, Brickley M. Surgical removal of third molars. Br Med J 1994 ; 309 : 620-1.

66 - Esposito M. Impacted wisdom teeth. Clin Evid 2006 ; $12: 1868-70$.

67 - Lee JT, Dodson TB. The effect of third molar presence and position on the risk of an angle fracture. J Oral Maxillofac Surg $2000 ; 58$ : 394-8.

68 - Mettes TG, Nienhuijs MEL, Van der Sanden WJM, Verdonschot $\mathrm{EH}$, Plasschaert AJM. Interventions for treating asymptomatic impacted wisdom teeth in ado- 
lescents and adults. Cochrane database of systematic reviews 2005, Issue 2 , art $\mathrm{N}^{\circ} \mathrm{CD} 003879$. DOI: 10.1002/14651858.CD003879.pub2.

69 - Mercier P, Precious D. Risks and benefits of removal of impacted third molars: a critical review of the literature. J Oral Maxillofac Surg 1992 ; 21 : 17-27.

70 - Tate TE. Impactions: observe or treat? J Calif Dent Assoc $1994 ; 22$ : 59-64.

71 - Brokaw WD. The third molar question: when and why should we recommend removal? Virginia Dent J $1991 ; 68$ : 18-21.

72 - American association of oral and maxillofacial surgeons. Public information - patient information - wisdom teeth, 2005.

73 - Kugelberg CF. Third molar surgery. Oral Maxillofac Surg Infect $1992 ; 2:$ 9-16.

74 - Lysell L, Rohun M. A study of indications used for removal of the mandibular third molar. Int $\mathrm{J}$ Oral Maxillofac Surg $1988 ; 17: 161-4$.

75 - Osborn T, Frederickson S, Small I, Togerson T. A prospective study of complications related to mandibular third molar surgery. J Oral Maxillofac Surg 1985 ; 43 : 767-9.

76 - Carmichael FA, McGowan DA. Incidence of nerve damage following third molar removal: a west of Scotland Oral Surgery research group study. Br J Oral Maxillofac Surg 1992 ; 30 : 78-82.

77 - Van der Linden W, Cleaton-Jones O, Lownie M. Diseases and lesions associated with third molars. Reviews of 1001 cases. Oral Surg Oral Med Oral Pathol Oral Radiol Endod 1995 ; 79 : 142-5.

78 - Von Wowern N, Nielsen HO. The fate of impacted lower third molars after the age of 20 . A four-year clinical follow-up. Int J Oral Maxillofac Surg 1989 ; 18 : 277-80.

79 - Stephens RG, Kogon SL, Reid JA. The unerupted or impacted third molar: a critical appraisal of its pathologic potential. Can Dent Assoc J 1989 ; 55 : 201-7.

80 - Song F, Landes DP, Glenny A-M, Sheldon TA. Prophylactic removal of impacted third molars: an assesment of published reviews. Br Dent J 1997 ; $182: 339-46$

81 - Tulloch JFC, Antczak AA, Wilkes JW. The application of decision analysis to evaluate the need for extraction of asymptomatic third molars. J Oral Maxillofac Surg 1987 ; 45 : 855-63.

82 - Jacquemart P. Dents de sagesse : faut-il les extraire? Service de presse du Congrès ADF, 2006.

83 - Worall SF. Dents de sagesse incluses ou enclavées. Décider pour traiter $2001 ; 1$ : 184-6.

84 - Brickley M, Kay E, Shepherd JP, Armstrong RA. Decision analysis for lower third molar surgery. Med Decis Making $1995 ; 15: 143-51$.

85 - Friedman JW. Containing the cost of third-molar extraction: a dilemma for health insurance. Public health Rep 1983 ; 98 : 376-84.
86 - Berge TI. Inability to work after surgical removal of mandibular third molars. Acta Odontol Scand 1997 ; $55: 64-9$.

87 - Carrigy JR. Early onset periodontal disease in Australian army recruits. MDSc thesis, University of Melbourne, 1997.

88 - Cowpe CB, Bell C. Audit for NHS: does the referral from GDPs requesting third molar extraction follow nice guideline. February 2002.

89 - Edwards MJ, Brickley MR, Goodey RD, Shepherd JP. The cost, effectiveness of removal and retention of asymptomatic, disease free third molars. Br Dent $\mathrm{J}$ $1999 ; 187: 380-4$

90 - Dhariwal DK, Goodey R, Shpherd JR. Trends in oral surgery in England and Wales 1991-2000. Br Dent J 2002 ; 192 : 639-45.

91 - Flick WG. Third molar controversy: framing the controversy as a public health issue. J Oral Maxillofac Surg $1999 ; 57: 438-44$.

92 - Girard P. Y a-t-il une dent de sagesse dans la mandibule ? Quintessence ADF, 2001. http://www.adf.asso.fr/cfm/site/thesaurus/detail: conference. $\mathrm{cfm}$ ? rubrique: origine $=47$ \& conference $=39 / 2001$.

93 - Mettes TG, Nienhuijs MEL, Van der Sanden WJM, Verdonschot EH, Plasschaert AJM. Interventions for treating asymptomatic impacted wisdom teeth in adolescents and adults. Cochrane Database of Systematic Reviews 2005, Issue 2. Art. No.: CD003879. DOI: 10.1002/14651858.CD003879. pub2.

94 - Nitzan DW, Tan O, Sela A. Pericoronitis: a reappraisal of its' clinical and microbiological aspects. J Oral Maxillofac Surg $1985 ; 43:$ 510-16.

95 - Knutsson K, Brehmer B, Lyseil L, Rohun M. Pathoses associated with mandibular third molars subjected to removal. Oral Surg Oral Med Oral Pathol Oral Radiol Endod 1996 ; 82 : 10-7.

96 - Brickley M, Shepherd J, Mancini G. Comparison of clinical treatment decisions with US National Institute of Health consensus indications for lower third molar removal. Br Dent J 1993 ; 175 : 102-5.

97 - Lopes V, Mumeyna R, Feinmann C, Harris M. Third molar surgery: an audit of the indications for surgery, post-operative complaints and patient satisfaction. Br Oral maxillofac Surg 1995 ; 33 : 33-5.

98 - Nordenram A, Hultin M, Kjellman O, Ramstrom G. Indications for surgical removal of mandibular third molar. Swed Dent J 1987 ; 11 : 23-9.

99 - NIH consensus development conference for removal of third molars. J of Oral Surg 1980 ; 38 : 235-6.

100 - Welch JT, Graves RW. Diagnosis, location and preoperative consultation for the difficult impaction. Dent Clin North Am 1979 ; 16 : 347-59.

101 - British standards institution. British standard glossary of dental terms.BSI, London, 1983. \begin{tabular}{l}
$\begin{array}{l}\text { médecine } \\
\text { buccale } \\
\text { chirurgie } \\
\text { buccale }\end{array}$ \\
\hline vOL. $14, N^{\circ} 4$ \\
2008 \\
\hline page 207
\end{tabular} 
102 - Piironen J, Ylipaavalniemi P. Local predisposing factors and clinical symptoms in pericoronatis. Proc Finn Dent Sc 1981; 77 : 278-82.

103 - Knutsson K, Brehmer B, Lyseli L, Rohlin M. General dental practitioners evaluation of the need for extraction of asymptomatic mandibular third molars. Community Dent Oral Epidemiol 1992 ; 20 : 347-50.

104 - Jones CM, O'Brien K, Blinkhorn AS, Rood JP. Dentist's agreement on treatment of asymptomatic impacted third molar teeth: interview study. Br Med J 1997 ; $315: 1204$.

105 - Huang H, Mercier P. Asymptomatic impacted teeth in edentulous jaws undergoing preprosthetic surgery. A long term evaluation. Int J Oral Maxillofac Surg $1992 ; 21: 147-9$.

106 - Hazelkorn HM, Macek MD. Perception of the need for removal of impacted third molars by general dentist and oral and maxillofacial surgeons. J Oral Maxillofac Surg 1994 ; 52 : 681-6.

107 - Lopes V, Memenya R, Feinmann C, Harris M. Third molar surgery: an audit of the indications for surgery, post-operative complaints and patient satisfaction. Br J Oral Maxillofac Surg 1995 ; 33 : 33-5.

médecine buccale chirurgie buccale

VOL. $14, \mathrm{~N}^{\circ} 4$ 2008

page 208
108 - Eliasson S, Heimdahl A. Pathological changes related to long-term impaction of third molars: a radiographic study. Int J Oral Maxillofac Surg 1989 ; 18 : 210-2.

109 - Van der Schoot EAM, Kuitert RB, Van Ginkel FC, Prahl-Andersen B. Clinical relevance of third permanent molars in relation to crowding after orthodontic treatment. J Dent $1997 ; 25: 167-9$.

110 - Lindqvist B, Thilander B. Extraction of third molars in case of anticipated crowding in the lower jaw. Am J Orthod $1982 ; 81: 130-9$.

111 - Ades A, Joondeph D, Little R, Chapkes M. A long term study of relationship of third molars to changes in the mandibular dental arch. Am J Orthod Dentofacial Orthop 1990 ; 97 : 323-35.

112 - Liedholm R, Knutsson K, Lysell L, Rohlin M, Brickley M, Shepherd J. Third molar treatment outcome: a comparison of patients' preferences in Sweden and Wales. Br Dent J 2005 ; 199 : 287-91.

113 -Department of defence instruction 6410.1. Standardization of dental classification and AR 4035.
114 - US army center for health promotion and preventive medicine. Directorate of health promotion and wellness. Readiness thru health 22-030-0307 - Oral fitness during deployment: dental caries.

115 - Richardson PS. Dental morbidity in United Kingdom armed forces, Iraq 2003. Milit Med 2005 ; 170 : 53641.

116 - Beth R. Deployment preparation: destination Iraq. Soldiers Magazine 2005,60 : 30-3.

117 - U.S. Army center for health promotion and preventive medicine - Directorate of health promotion and wellness. Readiness thru health 22-031-0307. Oral fitness during deployment: wisdom teeth.

118 - US Army Medical Activity - Alaska. Dentac - Alaska wellness guide - 2007.

119 - Tinler LE, Osbon DB, Lilly GE, Salem JE, Cutcher JL. Maxillo-facial injuries sustained in the Vietnam conflict. Mil Med 1974 ; 139 : 668-72.

120 - Bohnstedt S. Contrary to popular wisdom. Air Force - the official newspaper of the royal Australian air force. http://www. defence.gov.au/news/raafnews/editions/4621/health/story01.htm (Accès le 18 avril 2008).

121 - Army regulation 40-35 du 2 août 2004. Dental readiness and community oral health projection.

122 - Army regulation 40-3 du 3 avril 2006. Medical, dental and veterinary care. Chapter 6: dental care.

123 - Gunepin M, Derache F. Approche militaire américaine de l'optimisation de la mise en condition dentaire des Forces. Accepté pour publication dans Médecine et Armées.

124 - Keys D, Bartold M. Periodontal conditions of relevance to the Australian defence force. ADF Health 2001; $1: 114-8$.

125 - Health Policy Directive n404. Indications for removal of third molars in the Australian defence force. Nov 1994.

126 - Mahoney GD. The operational dental officer in the ADF. ADF Health $2003 ; 4: 40-4$.

127 - Note n`988/DEF/DCSSA/AST/TEC/1 du 21 avril 1994 relative à l'aptitude «dentaire » à servir Outre-mer ou en opération extérieure.

128 - Note n³98/DEF/DCSSA/AST/TEC/1 du 6 février 1995 relative à l'avulsion des dents de sagesse et aptitude " dentaire » à servir outre-mer ou en opération extérieure. 